\title{
A convenient synthesis of new amino acid-coupled benzanilides
}

\author{
S. M. EI Rayes, ${ }^{a^{*}}$ Ibrahim A. I. Ali, ${ }^{a}$ and Walid Fathalla ${ }^{b}$ \\ ${ }^{a}$ Department of Chemistry, Faculty of Science, Suez Canal University, Ismailia, Egypt \\ ${ }^{b}$ Department of Mathematical and Physical Sciences, Faculty of Engineering, \\ Suez Canal University, Port Said, Egypt \\ E-mail:samir_elrayes@yahoo.com
}

\section{Dedicated to the memory of Prof. Rajaa Basha}

\begin{abstract}
Methyl $N$-[2-(benzoylamino)benzoyl]-2-aminoalkanoates 4a-d were efficiently prepared by DCC coupling of 2-benzoylamino-benzoic acid 1 with amino acid methyl esters; or alternatively by reaction of amino acid esters with 2-phenyl-4H-benzo[d][1,3]oxazin-4-one (2). Dipeptides 7a-k were subsequently prepared following the azide-coupling method from esters 4 .
\end{abstract}

Keywords: Antibiotics, TCS, amino acids, dipeptide, DCC and azide coupling, benzanilides, 1,3-benzoxazinone

\section{Introduction}

Globally, multidrug-resistant bacteria are a major health problem leading to severe consequences. Nosocomial infections (super bug) have devastating effects with increased morbidity and mortality. New generations of antibiotics are produced to widen the spectrum of bactericidal effects. Also, multiple mechanisms of bactericidal action have been introduced. Recently, blockage of some gene transcription in bacteria via two-component system (TCS) inhibition was of major interest. ${ }^{1}$

Salicylanilides have shown antimycobacterial activity against classical mycobacterium tuberculosis H37Rv. They were proved to inhibit the bacterial TCS. ${ }^{2-6}$ Highly active salicylanilides has been produced as potent prodrugs; improving its solubility and low toxicity (Figure 1). ${ }^{7}$ Also, $N$-substituted carboxamide isothiazoles (ITF, denotivir) produced a remarkable immunotropic, antiviral and anti-inflammatory activities. ${ }^{8,9}$

$N$-Acylamino benzamides constitute an important class of biologically attractive compounds currently used as potent insecticides (Figure 1). ${ }^{10-12}$ The structure of all these compounds 
involves the presence of two phenyl rings linked to a spacer unit and two hydrogen bond donor sites. This spacer unit can be represented by a heterocyclic ring or by an acyclic moiety.

In this paper, we described the development of a new series of benzanilide derivatives, whose chemical modifications include N-terminal coupled amino acid and dipeptide derivatives to 2benzoylamino-benzoic acid, which can be used as active antibiotics.

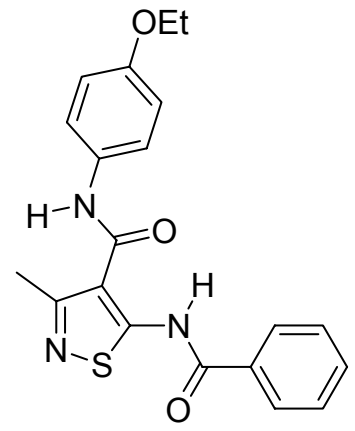

ITF<smiles>Cc1nsc(NC(=O)c2ccccc2)c1C(=O)Nc1ccc(Cl)cc1</smiles>

Denotivir<smiles>[R]C([R])([R])[C@@]([R])(NC)C(=O)Oc1ccc(Cl)cc1C(=O)N[14CH3]</smiles>

salicylanilides<smiles>[R]NC(=O)c1ccccc1NC(=O)c1ccccc1</smiles>

(N-Acylamino) benzamides

Figure 1. Biologically active carboxamides.

\section{Results and Discussion}

The synthesis of biologically active amino acid coupled derivatives was considered to be of major interest; particularly, methyl $N$-[2-(benzoylamino)benzoyl]-2-aminoalkanoates 4.

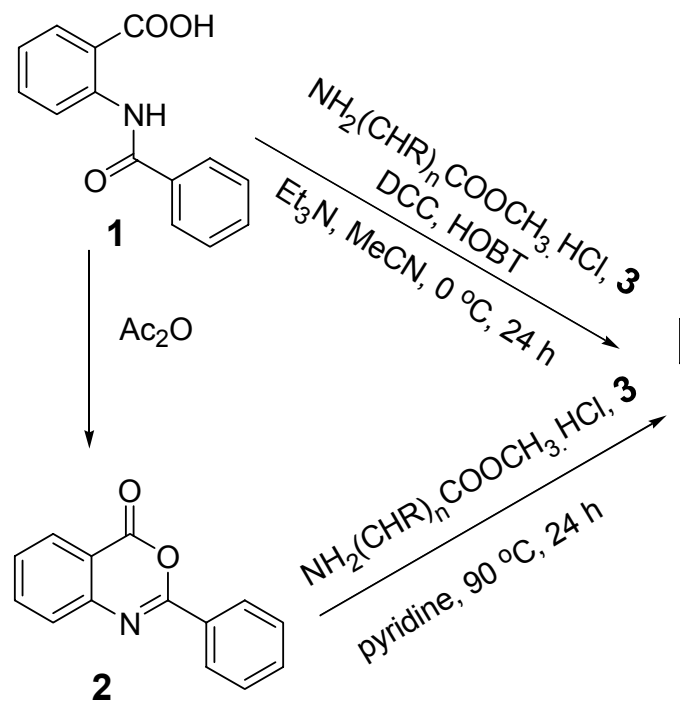

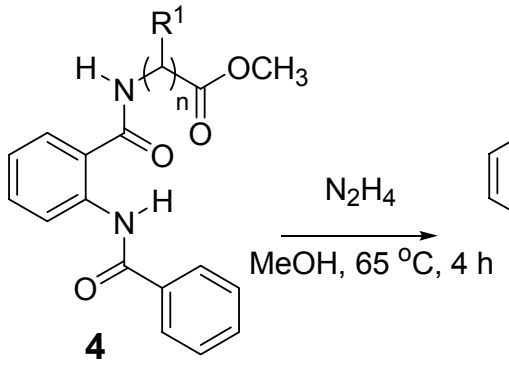

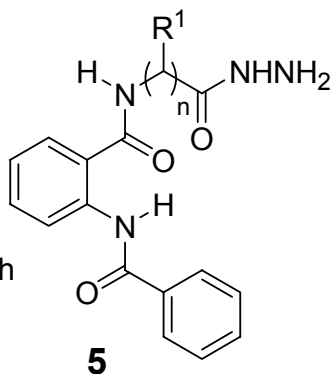

\begin{tabular}{l|cl}
$\mathbf{4 , 5}$ & $\mathrm{n}$ & $\mathrm{R}^{1}$ \\
\hline $\mathbf{a}$ (Gly) & 1 & $\mathrm{H}$ \\
$\mathbf{b}$ (L-Ala) & 1 & $\mathrm{CH}_{3}$ \\
$\mathbf{c}(\beta-A l a)$ & 2 & $\mathrm{H}$ \\
$\mathbf{d}$ (L-Ser) & 1 & $\mathrm{CH} 2 \mathrm{OH}$
\end{tabular}

\section{Scheme 1}


Treatment of 2-phenyl-4H-benzo[ $d][1,3]$ oxazin-4-one (2) with amino acid esters hydrochloride 3 in pyridine at $90{ }^{\circ} \mathrm{C}$ for $24 \mathrm{~h}$ gave a low yield of the acyclic amino acid ester derivatives 4a-d. ${ }^{13}$ Surprisingly, multiple changes in the reaction conditions (more reaction time at elevated temperature and evaporation of pyridine under reduced pressure rather than aqueous workup) did not improve the yield of this reaction. This low yield could be explained by the reversibility of the reaction; induced by the oxygen attack at the peptide bond with elimination of amino acid.

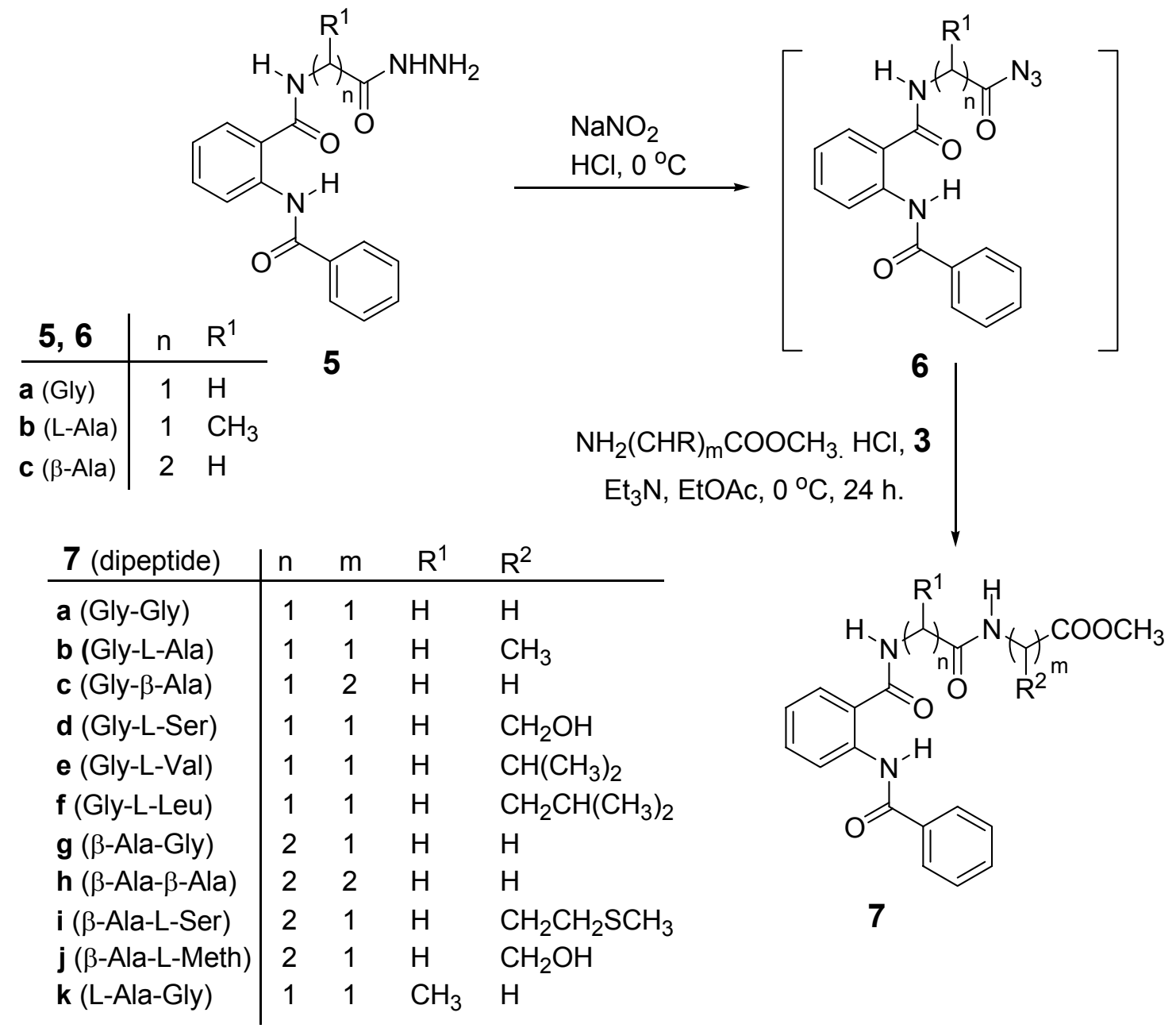

\section{Scheme 2}

However, the acyclic amino acid esters 4a-d were alternatively prepared via the DCC coupling method and showed an improvement in the yield (Scheme 1). The DCC coupling is one of the major tools employed in literature to introduce peptide bonds by the reaction of acid with amino acid methyl ester. Moreover, hydroxybenzotriazole (HOBT) is widely used as an additive to decrease racemization in the carbodiimide peptide coupling. ${ }^{14-16}$ Thus, treatment of acid 1 with the amino acid esters hydrochloride 3 in presence of coupling reagents DCC and HOBt at 0-25 
${ }^{\circ} \mathrm{C}$ in acetonitrile afforded amino acid esters 4a-d in 40-52 \% yield. Unfortunately, benzoxazinone 2 was also isolated from the reaction mixture as a by-product; which passively affected the yield of the reaction. This method has the advantage of a multi-component reaction with an overall moderate to good yield from easily available acid derivative $\mathbf{1}$ at low temperature to minimize the degree of racemization in amino acid coupling. The alternative reaction of benzoxazine in pyridine requires higher temperature and longer reaction time $\left(90{ }^{\circ} \mathrm{C}, 24 \mathrm{~h}\right)$ to produce 4 . Thus, both reactions reported herein are useful, due to their operational simplicity and availability.

Syntheses of the target dipeptide derivatives 7a-k were efficiently formed from the key intermediate esters 4 . The azide coupling method ${ }^{15,16}$ was reported to minimize the degree of racemization of amino acid coupling. The synthesis of dipeptide 7a-k via azide-coupling method is shown in Schemes 1 and 2. The esters 4a-d were boiled with hydrazine hydrate in methyl alcohol to produce hydrazides 5a-d. The corresponding hydrazides $\mathbf{5}$ were subsequently converted into azides 6; by treatment with $\mathrm{NaNO}_{2}$ and $\mathrm{HCl}$ mixture. The in situ generated azides solution $\mathbf{6}$ in ethyl acetate reacted with amino acid methyl esters hydrochloride $\mathbf{3}$ in the presence of triethylamine produced dipeptide derivatives 7a-k in good yield (Scheme 2).

The structure assignments of the amino acid esters 4a-d, hydrazides 5a-d and dipeptides 7a-k are based on spectral and physicochemical analysis (Figure 2).
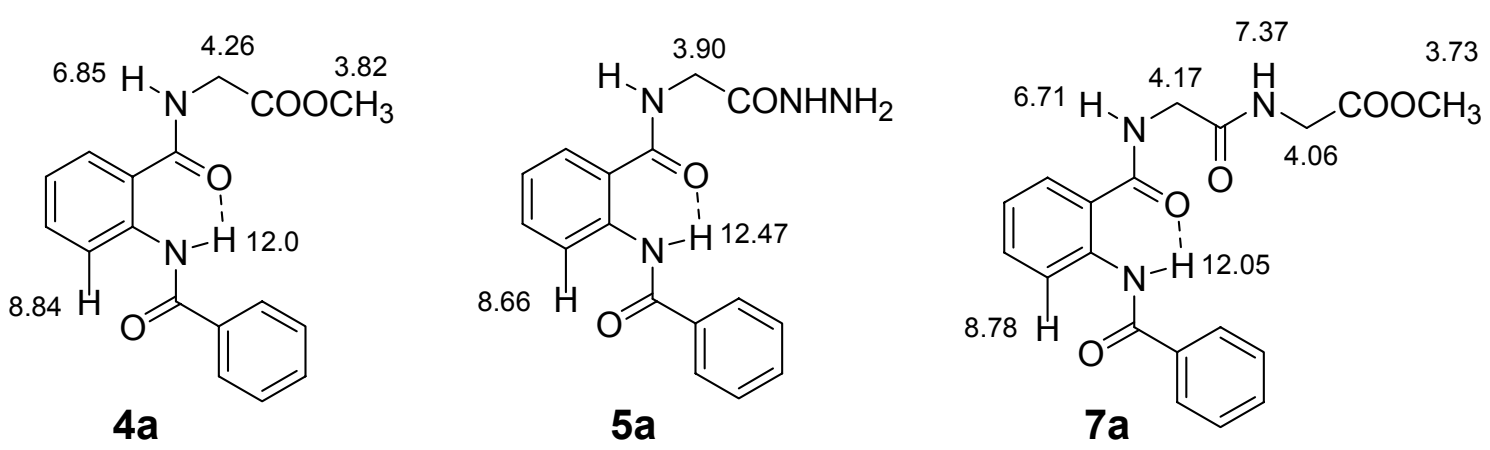

Figure 2. Selected ${ }^{1} \mathrm{H}$ NMR of compounds 4, 5 and 7.

All isolated products exhibited a rather interesting conformation as indicated from all ${ }^{1} \mathrm{H}$ NMR spectra, figure 2. Thus, the ${ }^{1} \mathrm{H}$ NMR spectrum of $4 \mathbf{a}$ showed an interesting exchangeable singlet signal at $\delta 12.0 \mathrm{ppm}$ corresponding to $\mathrm{NH}$ group. This implies that the $\mathrm{NH}$ group participate in an intramolecular hydrogen bond interaction of the type $\mathrm{N}-\mathrm{H} \cdots \mathrm{O}=\mathrm{C}$. ${ }^{9}$ Also, the ${ }^{1} \mathrm{H}$ NMR spectrum of $4 \mathbf{a}$ showed an aromatic proton at $\delta 8.84 \mathrm{ppm}$. Additionally, a broad singlet signals at $6.85 \mathrm{ppm}$ attributed to proton $\mathrm{H} 3$ of the phenyl ring and $\mathrm{NH}$ group of the glycine residue, respectively. The high down field chemical shift of the aromatic hydrogen implies an anisotropy caused by the adjacent carbonyl group. Both hydrogen bond interaction and this anisotropy are present in all isolated products; which indicates fixed conformation of the basic 
acyclic skeleton. The ${ }^{1} \mathrm{H}$ NMR spectrum of dipeptide 7a has shown a singlet, a triplet and a triplet signals at $\delta 12.05,7.37$ and $6.71 \mathrm{ppm}$. This was attributed to three NH groups as shown in figure 2. Also, the ${ }^{1} \mathrm{H}$ NMR spectrum of 7a gave three doublet signals at $\delta 4.06,4.17$ and 8.78 ppm consequent to two $\mathrm{CH}_{2}$ groups from the glycine residue and aromatic proton $\mathrm{H} 3$, respectively.

\section{Experimental Section}

General procedures. Solvent were purified and dried in the usual way. The boiling range of the petroleum ether used was $40-60{ }^{\circ} \mathrm{C}$. Thin layer chromatography (TLC): silica gel $60 \mathrm{~F}_{254}$ plastic plates (E. Merck, layer thickness $0.2 \mathrm{~mm}$ ) detected by UV absorption.

Elemental analyses were performed on a Flash EA-1112 instrument. at the Microanalytical laboratory, Faculty of Science, Suez Canal University, Ismailia, Egypt. Melting points were determined on a Buchi 510 melting-point apparatus and the values are uncorrected. NMR spectra measured with Bruker (300 MHz) and TMS (0.00 ppm) was used as internal standard. The starting compounds $\mathbf{1}$ and $\mathbf{2}$ were prepared according to described methods. ${ }^{17-18}$

\section{General procedure for preparation of compounds 4. Method A}

To a solution of 2-phenyl-4H-benzo[d][1,3] oxazin-4-one $(0.22 \mathrm{~g}, 1.0 \mathrm{mmol})$ in pyridine $(10 \mathrm{~mL})$, amino acid methyl ester hydrochloride $3(1.0 \mathrm{mmol})$ was added. The reaction mixture was refluxed at $90{ }^{\circ} \mathrm{C}$ for $24 \mathrm{~h}$. The resultant reddish solution was triturated portion wise with ice-cold water. The precipitated crystals of 4 were collected by filtration and crystallized from ethanol. Method B. To a cold solution $\left(-5^{\circ} \mathrm{C}\right)$ of amino acid methyl ester hydrochloride $3(1.0 \mathrm{mmol})$ and triethyl amine $(0.14 \mathrm{~mL}, 1.0 \mathrm{mmol})$ in acetonitrile $(6 \mathrm{~mL})$ was added 2-Benzoylamino-benzoic acid (1) (0.24 g, $1.0 \mathrm{mmol})$, dicyclohexylcarbodiimide (DCC) (0.21 g, $1.0 \mathrm{mmol})$ and hydroxybenzotriazole (HOBT) $(0.14 \mathrm{~g}, 1.0 \mathrm{mmol})$, successively. The reaction mixture was stirred at $0{ }^{\circ} \mathrm{C}$ for one hour, at $5{ }^{\circ} \mathrm{C}$ for one hour and at room temperature for 8 hours. The reaction mixture was set aside overnight. The precipitated dicyclohexyl urea was filtered off and the filtrate was evaporated under reduced pressure. The residue was purified by silica gel column chromatography eluted with EtOAc/ petroleum ether 1:4 to give pure 4.

Methyl $\boldsymbol{N}$-[2-(benzoylamino)benzoyl]-2-aminoacetate (4a). From $\mathrm{GlyOCH}_{3} \cdot \mathrm{HCl} \mathbf{3 a}(0.13 \mathrm{~g})$. Method A, colorless crystals (0.1 g, $32 \%$ ); mp 156-157 ${ }^{\circ} \mathrm{C} .{ }^{1} \mathrm{H}$ NMR (200 $\left.\mathrm{MHz}, \mathrm{CDCl}_{3}\right): \delta$ $12.00\left(1 \mathrm{H}, \mathrm{s}, \mathrm{D}_{2} \mathrm{O}\right.$ exchangeable, $\left.\mathrm{NH}\right), 8.84(1 \mathrm{H}, \mathrm{d}, J 8.2 \mathrm{~Hz}, \mathrm{ArH}), 8.02(2 \mathrm{H}, \mathrm{t}, J 7.8 \mathrm{~Hz}, \mathrm{ArH})$, 7.56-7.26 (5H, m, ArH), $7.13(1 \mathrm{H}, \mathrm{t}, J 7.8 \mathrm{~Hz}, \mathrm{ArH}), 6.85\left(1 \mathrm{H}, \mathrm{bs}, \mathrm{D}_{2} \mathrm{O}\right.$ exchangeable, $\left.\mathrm{NH}\right), 4.26$ $\left(2 \mathrm{H}, \mathrm{d}, J 5.0 \mathrm{~Hz}, \mathrm{CH}_{2}\right) 3.82\left(3 \mathrm{H}, \mathrm{s}, \mathrm{OCH}_{3}\right)$. Anal. Calcd. For $\mathrm{C}_{17} \mathrm{H}_{16} \mathrm{~N}_{2} \mathrm{O}_{4}$ (312.3): C, 65.38; H, 5.16; N, 8.97; Found: C, 65.42; H, 5.15; N, 9.01.

Method B, From 1 and GlyOMe.HCl 3a (0.14 g, 45 \%).

Methyl $\boldsymbol{N}$-[2-(benzoylamino)benzoyl]-2-aminopropanoate (4b). From $\mathrm{L}-\mathrm{AlaOCH} \cdot \mathrm{HCl} \mathbf{3 b}$ (0.14 g). Method A, colorless crystals (0.07 g, $21 \%$; mp 119-120 ${ }^{\circ} \mathrm{C} .{ }^{1} \mathrm{H}$ NMR $(200 \mathrm{MHz}$, 
$\left.\mathrm{CDCl}_{3}\right): \delta 12.02(1 \mathrm{H}, \mathrm{s}, \mathrm{NH}), 8.82(1 \mathrm{H}, \mathrm{d}, J 8.2 \mathrm{~Hz}, \mathrm{ArH}), 8.02(2 \mathrm{H}, \mathrm{t}, J 7.8 \mathrm{~Hz}, \mathrm{ArH}), 7.61-$ $7.49(5 \mathrm{H}, \mathrm{m}, \mathrm{ArH}), 7.12(1 \mathrm{H}, \mathrm{t}, J 7.8 \mathrm{~Hz}, \mathrm{ArH}), 6.96(1 \mathrm{H}, \mathrm{d}, J 6.0 \mathrm{~Hz}, \mathrm{NH}), 4.83-4.76(1 \mathrm{H}, \mathrm{m}$, $\mathrm{CH}), 3.80\left(3 \mathrm{H}, \mathrm{s}, \mathrm{OCH}_{3}\right), 1.56\left(3 \mathrm{H}, \mathrm{d}, J\right.$ 7.4, $\left.\mathrm{CH}_{3}\right)$. Anal. Calcd. For $\mathrm{C}_{18} \mathrm{H}_{18} \mathrm{~N}_{2} \mathrm{O}_{4}$ (326.4): C, 66.25; H, 5.56; N, 8.58; Found C, 66.32; H, 5.65; N, 8.66.

Method B, From 1 and L-AlaOCH $3 \cdot \mathrm{HCl} 3 \mathbf{b}(0.13 \mathrm{~g}, 40 \%)$.

Methyl $N$-[2-(benzoylamino)benzoyl]-3-aminopropanoate (4c). Method A. From $\beta$ $\mathrm{AlaOCH}_{3} \cdot \mathrm{HCl} 3 \mathrm{c}(0.14 \mathrm{~g})$. Method A, colorless crystals $(0.12 \mathrm{~g}, 36 \%)$; mp 102-103 ${ }^{\circ} \mathrm{C} .{ }^{1} \mathrm{H}$ NMR (200 MHz, $\left.\mathrm{CDCl}_{3}\right): \delta 12.16\left(1 \mathrm{H}, \mathrm{s}, \mathrm{D}_{2} \mathrm{O}\right.$ exchangeable, $\left.\mathrm{NH}\right), 8.82(1 \mathrm{H}, \mathrm{d}, J 8.2 \mathrm{~Hz}, \mathrm{ArH})$, $8.02(2 \mathrm{H}, \mathrm{t}, J 7.8 \mathrm{~Hz}, \mathrm{ArH}), 7.77\left(1 \mathrm{H}, \mathrm{bs}, \mathrm{D}_{2} \mathrm{O}\right.$ exchangeable, $\left.\mathrm{NH}\right), 7.57-7.49$ (5H, m, ArH), 7.10 $(1 \mathrm{H}, \mathrm{t}, J 7.8 \mathrm{~Hz}, \mathrm{ArH}), 3.78-3.70\left(5 \mathrm{H}, \mathrm{m}, \mathrm{CH}_{2}, \mathrm{OCH}_{3}\right), 2.67\left(2 \mathrm{H}, \mathrm{t}, J 5.4 \mathrm{~Hz}, \mathrm{CH}_{2}\right)$. Anal. Calcd. For $\mathrm{C}_{18} \mathrm{H}_{18} \mathrm{~N}_{2} \mathrm{O}_{4}$ (326.4): C, 66.25; H, 5.56; N, 8.58; Found C, 66.45; H, 5.64; N, 8.82.

Method B, From 1 and $\beta-\mathrm{AlaOCH}_{3} \cdot \mathrm{HCl} 3 \mathrm{c}(0.17 \mathrm{~g}, 52 \%)$.

Methyl $N$-[2-(benzoylamino)benzoyl]-2-amino-3-hydroxypropanoate (4d). From L$\mathrm{SerOCH}_{3} \cdot \mathrm{HCl} 3 \mathrm{dd}(0.16 \mathrm{~g})$. Method A, colorless crystals $(0.1 \mathrm{~g}, 29 \%)$; mp $178-179{ }^{\circ} \mathrm{C} .{ }^{1} \mathrm{H}$ NMR $\left(200 \mathrm{MHz}, \mathrm{CDCl}_{3}\right): \delta 11.96\left(1 \mathrm{H}, \mathrm{s}, \mathrm{D}_{2} \mathrm{O}\right.$ exchangeable, $\left.\mathrm{NH}\right), 8.82(1 \mathrm{H}, \mathrm{d}, J 8.2 \mathrm{~Hz}, \mathrm{ArH}), 8.00$ $(2 \mathrm{H}, \mathrm{t}, J 7.8 \mathrm{~Hz}, \mathrm{ArH}), 7.58-7.49$ (5H, m, ArH), 7.19 (1H, d, J 7.8 Hz, $\mathrm{D}_{2} \mathrm{O}$ exchangeable, $\left.\mathrm{NH}\right)$, 7.15 (1H, t, $J$ 7.8, ArH), 4.91-4.87 (1H, m, CH), $4.11\left(2 \mathrm{H}, \mathrm{dd}, J 3.0 \mathrm{~Hz}, J 8.0 \mathrm{~Hz}, \mathrm{CH}_{2}\right), 3.84$ $\left(3 \mathrm{H}, \mathrm{s}, \mathrm{OCH}_{3}\right), 2.31(1 \mathrm{H}, \mathrm{bs}, \mathrm{OH})$. Anal. Calcd. For $\mathrm{C}_{18} \mathrm{H}_{18} \mathrm{~N}_{2} \mathrm{O}_{5}(342.4): \mathrm{C}, 63.15 ; \mathrm{H}, 5.30 ; \mathrm{N}$, 8.18; Found C, 63.33; H, 5.41; N, 8.46.

Method B, From 1 and L-SerOCH $3 \cdot \mathrm{HCl} 3 d(0.14 \mathrm{~g}, 42 \%)$.

\section{General procedure for preparation of hydrazides 5a-d}

To a solution of esters 4a-d $(1.0 \mathrm{mmol})$ in methyl alcohol $(30 \mathrm{~mL})$ was added hydrazine hydrate $(0.24 \mathrm{~mL}, 5.0 \mathrm{mmol})$. The reaction mixture was refluxed for $4 \mathrm{~h}$, cooled and the precipitated white precipitate was filtered and crystallized from $\mathrm{MeOH}$.

2-Benzoylamino- $\boldsymbol{N}$-hydrazinocarbonylmethyl-benzamide (5a). Colorless crystals (0.29 g, 92 \%); mp 182-183 ${ }^{\circ} \mathrm{C} .{ }^{1} \mathrm{H}$ NMR (200 MHz, DMSO): $\delta 12.47\left(1 \mathrm{H}, \mathrm{s}, \mathrm{D}_{2} \mathrm{O}\right.$ exchangeable, $\left.\mathrm{NH}\right), 9.21$ $\left(1 \mathrm{H}, \mathrm{bs}, \mathrm{D}_{2} \mathrm{O}\right.$ exchangeable, $\left.\mathrm{NH}\right), 9.14\left(1 \mathrm{H}, \mathrm{bs}, \mathrm{D}_{2} \mathrm{O}\right.$ exchangeable, $\left.\mathrm{NH}\right), 8.66(1 \mathrm{H}, \mathrm{d}, J 8.4 \mathrm{~Hz}$, ArH), 7.97-7.90 (3H, m, ArH), 7.63-7.57 (4H, m, ArH), 7.15 (1H, t, J 7.8 Hz, ArH), 4.28 (2H, bs, $\mathrm{D}_{2} \mathrm{O}$ exchangeable, $\left.\mathrm{NH}_{2}\right), 3.90\left(2 \mathrm{H}, \mathrm{d}, J 6.0 \mathrm{~Hz}, \mathrm{CH}_{2}\right)$. Anal. Calcd. For $\mathrm{C}_{16} \mathrm{H}_{16} \mathrm{~N}_{4} \mathrm{O}_{3}(312.3)$ : C, 61.53; H, 5.16; N, 17.94; Found C, 61.71; H, 5.36; N, 18.12.

2-Benzoylamino- $\boldsymbol{N}$-(1-hydrazinocarbonyl-ethyl)-benzamide (5b). Colorless crystals $(0.27 \mathrm{~g}$, $84 \%$ ); mp 194-195 ${ }^{\circ} \mathrm{C} .{ }^{1} \mathrm{H}$ NMR (200 MHz, DMSO): $\delta 12.29\left(1 \mathrm{H}, \mathrm{s}, \mathrm{D}_{2} \mathrm{O}\right.$ exchangeable, $\mathrm{NH}$ ), $9.24\left(1 \mathrm{H}, \mathrm{bs}, \mathrm{D}_{2} \mathrm{O}\right.$ exchangeable, $\left.\mathrm{NH}\right), 8.88\left(1 \mathrm{H}, \mathrm{d}, J 6.8 \mathrm{~Hz}, \mathrm{D}_{2} \mathrm{O}\right.$ exchangeable, $\left.\mathrm{NH}\right), 8.66(1 \mathrm{H}$, $\mathrm{d}, J 8.2 \mathrm{~Hz}, \mathrm{ArH}), 7.97-7.93(3 \mathrm{H}, \mathrm{m}, \mathrm{ArH}), 7.62-7.56(4 \mathrm{H}, \mathrm{m}, \mathrm{ArH}), 7.23$ (1H, t, J 7.8 Hz, ArH), 4.54-4.48 (1H, m, CH), $4.31\left(2 \mathrm{H}, \mathrm{bs}, \mathrm{D}_{2} \mathrm{O}\right.$ exchangeable, $\left.\mathrm{NH}_{2}\right), 1.36\left(3 \mathrm{H}, \mathrm{d}, J 7.4 \mathrm{~Hz}, \mathrm{CH}_{3}\right)$. Anal. Calcd. For $\mathrm{C}_{17} \mathrm{H}_{18} \mathrm{~N}_{4} \mathrm{O}_{3}$ (326.4): C, 62.57; H, 5.56; N, 17.17; Found C, 62.67; H, 5.67; N, 17.39 .

2-Benzoylamino- $\boldsymbol{N}$-(2-hydrazinocarbonyl-ethyl)-benzamide (5c). Colorless crystals $(0.31 \mathrm{~g}$, $95 \%$ ); mp 208-209 ${ }^{\circ} \mathrm{C} .{ }^{1} \mathrm{H}$ NMR (200 MHz, DMSO): $\delta 12.62\left(1 \mathrm{H}, \mathrm{s}, \mathrm{D}_{2} \mathrm{O}\right.$ exchangeable, $\left.\mathrm{NH}\right)$, 
$9.08\left(1 \mathrm{H}, \mathrm{bs}, \mathrm{D}_{2} \mathrm{O}\right.$ exchangeable, $\left.\mathrm{NH}\right), 8.96\left(1 \mathrm{H}, \mathrm{d}, J 6.8 \mathrm{~Hz}, \mathrm{D}_{2} \mathrm{O}\right.$ exchangeable, $\left.\mathrm{NH}\right), 8.68(1 \mathrm{H}$, d, $J 8.6 \mathrm{~Hz}, \mathrm{ArH}), 7.98-7.95$ (2H, m, ArH), 7.84 (1H, d, J 8.2, ArH), 7.64-7.55 (4H, m, ArH), $7.21(1 \mathrm{H}, \mathrm{t}, J 7.8 \mathrm{~Hz}, \mathrm{ArH}), 4.23\left(2 \mathrm{H}, \mathrm{bs}, \mathrm{D}_{2} \mathrm{O}\right.$ exchangeable, $\left.\mathrm{NH}_{2}\right), 3.61-3.51\left(2 \mathrm{H}, \mathrm{m}, \mathrm{CH}_{2}\right)$, $2.37\left(2 \mathrm{H}, \mathrm{t}, J 7.2 \mathrm{~Hz}, \mathrm{CH}_{2}\right)$. Anal. Calcd. For $\mathrm{C}_{17} \mathrm{H}_{18} \mathrm{~N}_{4} \mathrm{O}_{3}$ (326.4): C, 62.57; H, 5.56; N, 17.17; Found C, 62.60; H, 5.51; N, 17.29.

2-Benzoylamino- $\mathbf{N}$-(1-hydrazinocarbonyl-2-hydroxy-ethyl)-benzamide $\quad$ (5d). Colorless crystals (0.26 g, $76 \%$ ); mp 198-199 ${ }^{\circ} \mathrm{C} .{ }^{1} \mathrm{H}$ NMR (200 MHz, DMSO): $\delta 12.42\left(1 \mathrm{H}, \mathrm{s}_{2} \mathrm{D}_{2} \mathrm{O}\right.$ exchangeable, $\mathrm{NH}), 9.27\left(1 \mathrm{H}, \mathrm{bs}, \mathrm{D}_{2} \mathrm{O}\right.$ exchangeable, $\left.\mathrm{NH}\right), 8.98\left(1 \mathrm{H}, \mathrm{bs}, \mathrm{D}_{2} \mathrm{O}\right.$ exchangeable, $\mathrm{NH}), 8.66$ (1H, d, J 8.6 Hz, ArH), 8.03-7.92 (3H, m, ArH), 7.65-7.51 (4H, m, ArH), 7.25 (1H, t, $J 7.8 \mathrm{~Hz}, \mathrm{ArH}), 4.94\left(1 \mathrm{H}, \mathrm{bs}, \mathrm{D}_{2} \mathrm{O}\right.$ exchangeable, $\left.\mathrm{OH}\right), 4.46-4.18\left(3 \mathrm{H}, \mathrm{m}, \mathrm{CH}, \mathrm{NH}_{2}\right), 3.52(2 \mathrm{H}, \mathrm{t}$, $J 6.2 \mathrm{~Hz}, \mathrm{CH}_{2}$ ). Anal. Calcd. For $\mathrm{C}_{17} \mathrm{H}_{18} \mathrm{~N}_{4} \mathrm{O}_{4}$ (342.1): C, 59.64; H, 5.30; N, 16.37; Found C, 59.51; H, 5.28; N, 16.31 .

\section{General procedure for preparation of dipeptides 7a-k}

To a cold solution $\left(-5^{\circ} \mathrm{C}\right)$ of hydrazide $5 \mathrm{a}-\mathrm{c}(1.0 \mathrm{mmol})$ in acetic acid $(6 \mathrm{ml}), 1 \mathrm{~N} \mathrm{HCl}(3 \mathrm{ml})$, and water $(25 \mathrm{ml})$ was added a solution of $\mathrm{NaNO}_{2}(0.87 \mathrm{~g}, 1.0 \mathrm{mmol})$ in cold water $(3 \mathrm{ml})$. The reaction mixture was stirred at $-5^{\circ} \mathrm{C}$ for $15 \mathrm{~min}$. The yellow syrup formed was extracted with cold ethyl acetate $(30 \mathrm{ml})$, washed with cold $3 \% \mathrm{NaHCO}_{3}, \mathrm{H}_{2} \mathrm{O}$ and finally dried $\left(\mathrm{Na}_{2} \mathrm{SO}_{4}\right)$. To this solution amino acid esters $3(1.0 \mathrm{mmol})$ in ethyl acetate $(20 \mathrm{~mL})$ containing $0.2 \mathrm{~mL}^{\circ} \mathrm{Et}_{3} \mathrm{~N}$ were added. The reaction mixture was kept at $-5{ }^{\circ} \mathrm{C}$ for $24 \mathrm{~h}$, then at $25{ }^{\circ} \mathrm{C}$ for another $24 \mathrm{~h}$. The solution was evaporated to dryness, and the residue was crystallized from petroleum ether/ ethyl acetate to give the desired product.

Methyl 2-\{[2-(Benzoylamino)benzoyl] amino\}-2`-(acetylamino)acetate (7a). From hydrazide 5a $(0.31 \mathrm{~g})$ and $\mathrm{GlyOCH}_{3} \cdot \mathrm{HCl} 3 \mathbf{a}(0.13 \mathrm{~g})$. Colorless crystals $(0.21 \mathrm{~g}, 58 \%) ; \mathrm{mp} 113-114{ }^{\circ} \mathrm{C} .{ }^{1} \mathrm{H}$ NMR $\left(200 \mathrm{MHz}, \mathrm{CDCl}_{3}\right): \delta 12.05\left(1 \mathrm{H}, \mathrm{s}, \mathrm{D}_{2} \mathrm{O}\right.$ exchangeable, $\left.\mathrm{NH}\right), 8.78(1 \mathrm{H}, \mathrm{d}, J 8.6, \operatorname{ArH})$, $8.00(2 \mathrm{H}, \mathrm{d}, J 8.4 \mathrm{~Hz}, \mathrm{ArH}), 7.62(1 \mathrm{H}, \mathrm{d}, J 8.0 \mathrm{~Hz}, \mathrm{ArH}), 7.57-7.49$ (4H, m, ArH), 7.37 (1H, t, $J$ $7.2 \mathrm{~Hz}, \mathrm{D}_{2} \mathrm{O}$ exchangeable, $\left.\mathrm{NH}\right), 7.10(1 \mathrm{H}, \mathrm{t}, J 7.6 \mathrm{~Hz}, \mathrm{ArH}), 6.71\left(1 \mathrm{H}, \mathrm{t}, J 7.4 \mathrm{~Hz}, \mathrm{D}_{2} \mathrm{O}\right.$ exchangeable, NH), $4.17\left(2 \mathrm{H}, \mathrm{d}, J 5.2 \mathrm{~Hz}, \mathrm{CH}_{2}\right), 4.06\left(2 \mathrm{H}, \mathrm{d}, J 5.4 \mathrm{~Hz}, \mathrm{CH}_{2}\right), 3.73$ (3H, s, $\mathrm{OCH}_{3}$ ). Anal. Calcd. For $\mathrm{C}_{19} \mathrm{H}_{19} \mathrm{~N}_{3} \mathrm{O}_{5}$ (369.4): C, 61.78; H, 5.18; N, 11.38; Found C, 61.81; H, $5.32 ; \mathrm{N}, 11.64$.

Methyl 2-\{[2-(Benzoylamino)benzoyl]amino\}-2`-(acetylamino)propanoate (7b). From hydrazide $5 \mathbf{a}(0.31 \mathrm{~g})$ and $\mathrm{L}-\mathrm{AlaOCH}_{3} \cdot \mathrm{HCl} \mathbf{3 b}(0.14 \mathrm{~g})$. Colorless crystals $(0.16 \mathrm{~g}, 41 \%)$; mp 115-116 ${ }^{\circ} \mathrm{C} .{ }^{1} \mathrm{H}$ NMR $\left(200 \mathrm{MHz}, \mathrm{CDCl}_{3}\right): \delta 12.06\left(1 \mathrm{H}, \mathrm{s}, \mathrm{D}_{2} \mathrm{O}\right.$ exchangeable, $\left.\mathrm{NH}\right), 8.76(1 \mathrm{H}, \mathrm{d}$, $J 8.6 \mathrm{~Hz}, \mathrm{ArH}), 8.00(2 \mathrm{H}, \mathrm{d}, J 7.6 \mathrm{~Hz}, \mathrm{ArH}), 7.62(1 \mathrm{H}, \mathrm{d}, J 8.6 \mathrm{~Hz}, \mathrm{ArH}), 7.59-7.42$ (5H, m, ArH, $\mathrm{NH}), 7.06(1 \mathrm{H}, \mathrm{t}, J 8.0 \mathrm{~Hz}, \mathrm{ArH}), 6.89\left(1 \mathrm{H}, \mathrm{d}, J 7.2 \mathrm{~Hz}, \mathrm{D}_{2} \mathrm{O}\right.$ exchangeable, $\left.\mathrm{NH}\right), 4.65-4.50(1 \mathrm{H}$, m, CH), $4.13\left(2 \mathrm{H}, \mathrm{d}, J 4.4 \mathrm{~Hz}, \mathrm{CH}_{2}\right), 3.69\left(3 \mathrm{H}, \mathrm{s}, \mathrm{OCH}_{3}\right), 1.38\left(3 \mathrm{H}, \mathrm{d}, J 7.0 \mathrm{~Hz}, \mathrm{CH}_{3}\right)$. Anal. Calcd. For $\mathrm{C}_{20} \mathrm{H}_{21} \mathrm{~N}_{3} \mathrm{O}_{5}$ (383.4): C, 62.65; H, 5.52; N, 10.96; Found C, 62.48; H, 5.41; N, 10.87.

Methyl 2-\{[2-(Benzoylamino)benzoyl]amino $\}-3$-(acetylamino) propanoate (7c). From hydrazide $5 \mathbf{a}(0.31 \mathrm{~g})$ and $\beta-\mathrm{AlaOCH}_{3} \cdot \mathrm{HCl} \mathbf{3 c}(0.14 \mathrm{~g})$. Colorless crystals $(0.27 \mathrm{~g}, 72 \%)$; $\mathrm{mp}$ 167-168 ${ }^{\circ} \mathrm{C} .{ }^{1} \mathrm{H}$ NMR $\left(200 \mathrm{MHz}, \mathrm{CDCl}_{3}\right): \delta 12.09\left(1 \mathrm{H}, \mathrm{s}, \mathrm{D}_{2} \mathrm{O}\right.$ exchangeable, $\left.\mathrm{NH}\right), 8.80(1 \mathrm{H}, \mathrm{d}$, 
$J$ 8.0, ArH), $8.00(2 \mathrm{H}, \mathrm{d}, J 7.2 \mathrm{~Hz}, \mathrm{ArH}), 7.63(1 \mathrm{H}, \mathrm{d}, J 7.6 \mathrm{~Hz}, \mathrm{ArH}), 7.56-7.44$ (5H, m, ArH, $\mathrm{NH}), 7.11(1 \mathrm{H}, \mathrm{t}, J 8.0 \mathrm{~Hz}, \mathrm{ArH}), 6.59\left(1 \mathrm{H}, \mathrm{bs}, \mathrm{D}_{2} \mathrm{O}\right.$ exchangeable, $\left.\mathrm{NH}\right), 4.09$ (2H, d, J 4.6 Hz, $\left.\mathrm{CH}_{2}\right), 3.65\left(3 \mathrm{H}, \mathrm{s}, \mathrm{OCH}_{3}\right), 3.57\left(2 \mathrm{H}, \mathrm{q}, J 6.2 \mathrm{~Hz}, \mathrm{CH}_{2}\right), 2.56\left(2 \mathrm{H}, \mathrm{t}, J 6.0 \mathrm{~Hz}, \mathrm{CH}_{2}\right)$. Anal. Calcd. For $\mathrm{C}_{20} \mathrm{H}_{21} \mathrm{~N}_{3} \mathrm{O}_{5}$ (383.4): C, 62.65; H, 5.52; N, 10.96; Found C, 62.51; H, 5.43; N, 10.93 .

Methyl 2-\{[2-(Benzoylamino)benzoyl]amino $\}-2$-(acetylamino)-3'-hydroxypropan-oate (7d). From hydrazide $5 \mathbf{a}(0.31 \mathrm{~g})$ and $\mathrm{L}-\mathrm{SerOCH}_{3} \cdot \mathrm{HCl} \mathbf{3 d}(0.16 \mathrm{~g})$. Colorless crystals $(0.22 \mathrm{~g}, 55 \%)$; mp 144-145 ${ }^{\circ} \mathrm{C} .{ }^{1} \mathrm{H}$ NMR $\left(200 \mathrm{MHz}, \mathrm{CDCl}_{3}\right): \delta 12.10\left(1 \mathrm{H}, \mathrm{s}, \mathrm{D}_{2} \mathrm{O}\right.$ exchangeable, $\left.\mathrm{NH}\right), 8.62$ $(1 \mathrm{H}, \mathrm{d}, J 8.6 \mathrm{~Hz}, \mathrm{ArH}), 8.16\left(1 \mathrm{H}, \mathrm{bs}, \mathrm{D}_{2} \mathrm{O}\right.$ exchangeable, $\left.\mathrm{NH}\right), 7.85-7.73(2 \mathrm{H}, \mathrm{m}, \mathrm{ArH}), 7.60(2 \mathrm{H}$, d, J 7.8 Hz, ArH), 7.35-7.04 (5H, m, ArH, NH), 6.96 (1H, t, J 7.6 Hz, ArH), 4.47-4.40 (1H, m, $\mathrm{CH}), 4.01-3.92\left(2 \mathrm{H}, \mathrm{m}, \mathrm{CH}_{2}\right), 3.67\left(2 \mathrm{H}, \mathrm{dd}, J 3.0 \mathrm{~Hz}, J 6.0 \mathrm{~Hz}, \mathrm{OCH}_{2}\right), 3.53\left(3 \mathrm{H}, \mathrm{s}, \mathrm{OCH}_{3}\right)$, 2.62 (1H, bs, OH). Anal. Calcd. For $\mathrm{C}_{20} \mathrm{H}_{21} \mathrm{~N}_{3} \mathrm{O}_{6}$ (399.4): C, 60.14; H, 5.30; N, 10.52; Found C, $60.43 ; \mathrm{H}, 5.57$; N, 10.66 .

Methyl 2-\{[2-(Benzoylamino)benzoyl]amino\}-2`(acetylamino)-3`-methylbutanoate (7e). From hydrazide $5 \mathbf{a}(0.31 \mathrm{~g})$ and $\mathrm{L}-\mathrm{ValOCH}_{3} \cdot \mathrm{HCl} \mathbf{3 e}(0.17 \mathrm{~g})$. Colorless crystals $(0.20 \mathrm{~g}, 49 \%)$; mp 165-166 ${ }^{\circ} \mathrm{C} .{ }^{1} \mathrm{H}$ NMR $\left(200 \mathrm{MHz}, \mathrm{CDCl}_{3}\right): \delta 12.11\left(1 \mathrm{H}, \mathrm{s}, \mathrm{D}_{2} \mathrm{O}\right.$ exchangeable, $\left.\mathrm{NH}\right), 8.79$ $(1 \mathrm{H}, \mathrm{d}, J 8.4 \mathrm{~Hz}, \mathrm{ArH}), 8.00(2 \mathrm{H}, \mathrm{d}, J 8.0 \mathrm{~Hz}, \mathrm{ArH}), 7.63(2 \mathrm{H}, \mathrm{d}, J 8.4 \mathrm{~Hz}, \mathrm{ArH}), 7.57-7.42(5 \mathrm{H}$, m, ArH, NH), $7.07(1 \mathrm{H}, \mathrm{t}, J 7.0 \mathrm{~Hz}, \mathrm{ArH}), 6.72\left(1 \mathrm{H}, \mathrm{d}, J 8.0 \mathrm{~Hz}, \mathrm{D}_{2} \mathrm{O}\right.$ exchangeable, NH), 4.64$4.48(1 \mathrm{H}, \mathrm{m}, \mathrm{CH}), 4.15\left(2 \mathrm{H}, \mathrm{d}, J 4.4 \mathrm{~Hz}, \mathrm{CH}_{2}\right), 3.70\left(3 \mathrm{H}, \mathrm{s}, \mathrm{OCH}_{3}\right), 1.21-1.08(1 \mathrm{H}, \mathrm{m}, \mathrm{CH}), 0.87$ $\left(6 \mathrm{H}, \mathrm{d}, J 6.0 \mathrm{~Hz}, 2 \mathrm{CH}_{3}\right)$. Anal. Calcd. For $\mathrm{C}_{22} \mathrm{H}_{25} \mathrm{~N}_{3} \mathrm{O}_{5}$ (411.5): C, 64.22; H, 6.12; N, 10.12; Found C, 64.18; H, 6.04; N, 10.08.

Methyl 2-\{[2-(Benzoylamino)benzoyl $]$ amino $\}-2$-(acetylamino)-4'-methylpentanoate (7f). From hydrazide $5 \mathbf{a}(0.31 \mathrm{~g})$ and $\mathrm{L}-\mathrm{LeuOCH}_{3} \cdot \mathrm{HCl} \mathbf{3 f}(0.18 \mathrm{~g})$. Colorless crystals $(0.22 \mathrm{~g}, 51 \%)$; mp 125-126 ${ }^{\circ} \mathrm{C} .{ }^{1} \mathrm{H}$ NMR $\left(200 \mathrm{MHz}, \mathrm{CDCl}_{3}\right): \delta 12.13\left(1 \mathrm{H}, \mathrm{s}, \mathrm{D}_{2} \mathrm{O}\right.$ exchangeable, $\left.\mathrm{NH}\right), 8.77$ $(1 \mathrm{H}, \mathrm{d}, J 8.2 \mathrm{~Hz}, \mathrm{ArH}), 7.99$ (2H, d, J 8.0 Hz, ArH), 7.62 (2H, d, J 7.0 Hz, ArH), 7.54-7.42 (4H, m, ArH, NH), $7.04(1 \mathrm{H}, \mathrm{t}, J 7.2 \mathrm{~Hz}, \mathrm{ArH}), 6.84\left(1 \mathrm{H}, \mathrm{d}, J 8.2 \mathrm{~Hz}, \mathrm{D}_{2} \mathrm{O}\right.$ exchangeable, NH), 4.67$4.52(1 \mathrm{H}, \mathrm{m}, \mathrm{CH}), 4.13\left(2 \mathrm{H}, \mathrm{d}, J 4.6 \mathrm{~Hz}, \mathrm{CH}_{2}\right), 3.66\left(3 \mathrm{H}, \mathrm{s}, \mathrm{OCH}_{3}\right), 1.65-1.46\left(2 \mathrm{H}, \mathrm{m}, \mathrm{CH}_{2}\right)$, 1.25-1.07 (1H, m, CH) $0.86\left(6 \mathrm{H}, \mathrm{d}, J 6.0 \mathrm{~Hz}, 2 \mathrm{CH}_{3}\right)$. Anal. Calcd. For $\mathrm{C}_{23} \mathrm{H}_{27} \mathrm{~N}_{3} \mathrm{O}_{5}$ (425.5): C, 64.93; H, 6.40; N, 9.88; Found C, 64.88; H, 6.37; N, 9.81.

Methyl 3-\{[2-(Benzoylamino)benzoyl]amino\}-2'-(propionylamino)acetate $\quad(8 \mathrm{~g})$. From hydrazide $5 \mathbf{c}(0.33 \mathrm{~g})$ and $\mathrm{GlyOCH}_{3} \cdot \mathrm{HCl} 3 \mathbf{3}(0.13 \mathrm{~g})$. Colorless crystals $(0.29 \mathrm{~g}, 77 \mathrm{\%})$; mp 158$159{ }^{\circ} \mathrm{C} .{ }^{1} \mathrm{H}$ NMR $\left(200 \mathrm{MHz}, \mathrm{CDCl}_{3}\right): \delta 12.26\left(1 \mathrm{H}, \mathrm{s}, \mathrm{D}_{2} \mathrm{O}\right.$ exchangeable, $\left.\mathrm{NH}\right), 8.81(1 \mathrm{H}, \mathrm{d}, J 8.2$ $\mathrm{Hz}, \mathrm{ArH}), 8.05$ (2H, d, J 8.2 Hz, ArH), 7.59-7.38 (5H, m, ArH, NH), $7.11(1 \mathrm{H}, \mathrm{t}, J 7.6 \mathrm{~Hz}, \mathrm{ArH})$, $6.11\left(1 \mathrm{H}, \mathrm{bs}, \mathrm{D}_{2} \mathrm{O}\right.$ exchangeable, $\left.\mathrm{NH}\right), 4.05\left(2 \mathrm{H}, \mathrm{d}, J 5.4 \mathrm{~Hz}, \mathrm{CH}_{2}\right), 3.82-3.74\left(5 \mathrm{H}, \mathrm{m}, \mathrm{CH}_{2}\right.$, $\left.\mathrm{OCH}_{3}\right), 2.59\left(2 \mathrm{H}, \mathrm{t}, J 5.6 \mathrm{~Hz}, \mathrm{CH}_{2}\right)$. Anal. Calcd. For $\mathrm{C}_{20} \mathrm{H}_{21} \mathrm{~N}_{3} \mathrm{O}_{5}(383.4)$ : C, 62.65; H, 5.52; N, 10.96; Found C, 62.72; H, 5.64; N, 11.21.

Methyl 3-\{[2-(Benzoylamino) benzoyl]amino\}-3`-(propionylamino)propanoate (7h). From hydrazide $5 \mathrm{c}(0.33 \mathrm{~g})$ and $\beta$-AlaOCH${ }_{3} \cdot \mathrm{HCl} 3 \mathrm{c}(0.14 \mathrm{~g})$. Colorless crystals $(0.32 \mathrm{~g}, 81 \%)$; mp 102-103 ${ }^{\circ} \mathrm{C} .{ }^{1} \mathrm{H}$ NMR $\left(200 \mathrm{MHz}, \mathrm{CDCl}_{3}\right): \delta 12.15\left(1 \mathrm{H}, \mathrm{s}, \mathrm{D}_{2} \mathrm{O}\right.$ exchangeable, $\left.\mathrm{NH}\right), 8.83(1 \mathrm{H}, \mathrm{d}$, $J 8.2 \mathrm{~Hz}, \mathrm{ArH}), 8.07-8.03(2 \mathrm{H}, \mathrm{m}, \mathrm{ArH}), 7.58-7.50(5 \mathrm{H}, \mathrm{m}, \mathrm{ArH}, \mathrm{NH}), 7.11(1 \mathrm{H}, \mathrm{t}, J 7.6 \mathrm{~Hz}$, ArH). $6.11\left(1 \mathrm{H}, \mathrm{bs}, \mathrm{D}_{2} \mathrm{O}\right.$ exchangeable, $\left.\mathrm{NH}\right), 3.82-3.69\left(5 \mathrm{H}, \mathrm{m}, \mathrm{CH}_{2}, \mathrm{OCH}_{3}\right), 3.53(2 \mathrm{H}, \mathrm{q}, J 6.4$ 
$\mathrm{Hz}, \mathrm{CH}_{2}$ ), 2.52-2.47 (4H, m, $2 \mathrm{CH}_{2}$ ). Anal. Calcd. For $\mathrm{C}_{21} \mathrm{H}_{23} \mathrm{~N}_{3} \mathrm{O}_{5}$ (397.4): C, 63.46; H, 5.83; N, 10.57; Found C, 63.55; H, 5.91; N, 10.69 .

Methyl 3-\{[2-(Benzoylamino)benzoyl]amino\}-2`-(propionylamino)3`-hydroxyprop-anoate (7i). From hydrazide 5c $(0.33 \mathrm{~g})$ and $\mathrm{L}_{-} \mathrm{SerOCH}_{3} \cdot \mathrm{HCl} \mathbf{3 d}(0.16 \mathrm{~g})$. Colorless crystals $(0.26 \mathrm{~g}, 63$ \%); mp 109-110 ${ }^{\circ} \mathrm{C} .{ }^{1} \mathrm{H}$ NMR (200 MHz, $\left.\mathrm{CDCl}_{3}\right): \delta 12.12\left(1 \mathrm{H}, \mathrm{s}, \mathrm{D}_{2} \mathrm{O}\right.$ exchangeable, $\left.\mathrm{NH}\right), 8.72$ $(1 \mathrm{H}, \mathrm{d}, J 8.2 \mathrm{~Hz}, \mathrm{ArH}), 8.02$ (2H, d, $J 8.2 \mathrm{~Hz}, \mathrm{ArH}), 7.59-7.47$ (5H, m, ArH, NH), 7.08 (1H, t, $J$ 7.6, $\mathrm{ArH}), 6.70\left(1 \mathrm{H}, \mathrm{d}, J 7.2 \mathrm{~Hz}, \mathrm{D}_{2} \mathrm{O}\right.$ exchangeable, $\left.\mathrm{NH}\right), 5.77\left(1 \mathrm{H}, \mathrm{bs}, \mathrm{D}_{2} \mathrm{O}\right.$ exchangeable, $\left.\mathrm{NH}\right)$, 4.65-4.61 (1H, m, CH), $3.97\left(2 \mathrm{H}, \mathrm{dd}, J 3.1 \mathrm{~Hz}, J 8.0 \mathrm{~Hz}, \mathrm{CH}_{2}\right), 3.75-3.65\left(5 \mathrm{H}, \mathrm{m}, \mathrm{CH}_{2}\right.$, $\left.\mathrm{OCH}_{3}\right), 2.78\left(1 \mathrm{H}, \mathrm{bs}, \mathrm{OH}, \mathrm{D}_{2} \mathrm{O}\right.$ exchangeable), $2.57\left(2 \mathrm{H}, \mathrm{t}, J 6.0 \mathrm{~Hz}, \mathrm{CH}_{2}\right)$. Anal. Calcd. For $\mathrm{C}_{21} \mathrm{H}_{23} \mathrm{~N}_{3} \mathrm{O}_{6}$ (413.4): C, 61.01; H, 5.61; N, 10.16; Found C, 61.36; H, 5.79; N 10.43.

Methyl 3-\{[2-(Benzoylamino)benzoyl]amino $\}$-2`(propionylamino)-4 ${ }^{`}$-methylsulfanyl butanoate (7j). From hydrazide $5 \mathbf{c}(0.33 \mathrm{~g})$ and $\mathrm{L}-\mathrm{MetOCH}_{3} \cdot \mathrm{HCl} \mathrm{3g}(0.20 \mathrm{~g})$. Colorless crystals $(0.21 \mathrm{~g}, 47 \%) ; \mathrm{mp} 148-149{ }^{\circ} \mathrm{C}$. ${ }^{1} \mathrm{H} \mathrm{NMR} \quad\left(200 \mathrm{MHz}, \mathrm{CDCl}_{3}\right): \delta 12.28\left(1 \mathrm{H}, \mathrm{bs}, \mathrm{D}_{2} \mathrm{O}\right.$ exchangeable, NH), $8.81(1 \mathrm{H}, \mathrm{d}, J 8.2 \mathrm{~Hz}, \mathrm{ArH}), 8.04(2 \mathrm{H}, \mathrm{d}, J 8.2 \mathrm{~Hz}, \mathrm{ArH}), 7.58-7.48(5 \mathrm{H}, \mathrm{m}$, ArH), $7.10(1 \mathrm{H}, \mathrm{t}, J 7.6 \mathrm{~Hz}, \mathrm{ArH}), 6.41\left(1 \mathrm{H}, \mathrm{bs}, \mathrm{D}_{2} \mathrm{O}\right.$ exchangeable, $\left.\mathrm{NH}\right), 6.24\left(1 \mathrm{H}, \mathrm{bs}, \mathrm{D}_{2} \mathrm{O}\right.$ exchangeable, $\mathrm{NH}), 4.77-4.71(1 \mathrm{H}, \mathrm{m}, \mathrm{CH}), 3.72-3.58\left(5 \mathrm{H}, \mathrm{s}, \mathrm{CH}_{2} \mathrm{~S}, \mathrm{OCH}_{3}\right), 3.53(2 \mathrm{H}, \mathrm{q}, J 6.4$ $\left.\mathrm{Hz}, \mathrm{NHCH}_{2}\right), 2.60-2.47\left(4 \mathrm{H}, \mathrm{m}, \underline{\mathrm{CH}}_{2} \mathrm{CO}, \mathrm{CHCH}_{2}\right), 2.05\left(3 \mathrm{H}, \mathrm{s}, \mathrm{SCH}_{3}\right)$. Anal. Calcd. For $\mathrm{C}_{23} \mathrm{H}_{27} \mathrm{~N}_{3} \mathrm{O}_{5} \mathrm{~S}$ (457.5): C, 60.38; H, 5.95; N, 9.18; Found C, 60.54; H, 6.11, N; 9.38.

Methyl 2-\{[2-(Benzoylamino)benzoyl]amino\}-2`-(propionylamino)acetate (7k). From hydrazide $5 \mathbf{b}(0.33 \mathrm{~g})$ and $\mathrm{GlyOCH}_{3} \cdot \mathrm{HCl} 3 \mathbf{a}(0.13 \mathrm{~g})$. Colorless crystals $(0.15 \mathrm{~g}, 40 \%)$; mp $125-$ $126{ }^{\circ} \mathrm{C} .{ }^{1} \mathrm{H}$ NMR $\left(200 \mathrm{MHz}, \mathrm{CDCl}_{3}\right): \delta 12.11\left(1 \mathrm{H}, \mathrm{s}, \mathrm{D}_{2} \mathrm{O}\right.$ exchangeable, $\left.\mathrm{NH}\right), 8.75(1 \mathrm{H}, \mathrm{d}, J 8.2$ $\mathrm{Hz}, \mathrm{ArH}), 8.06$ (2H, d, J 7.6 Hz, ArH), 7.64-7.52 (5H, m, ArH, NH), 7.15 (1H, t, J 8.0 Hz, ArH), $7.05\left(1 \mathrm{H}, \mathrm{d}, J 7.2 \mathrm{~Hz}, \mathrm{D}_{2} \mathrm{O}\right.$ exchangeable, $\left.\mathrm{NH}\right), 5.53\left(1 \mathrm{H}, \mathrm{d}, J 7.2 \mathrm{~Hz}, \mathrm{D}_{2} \mathrm{O}\right.$ exchangeable, $\left.\mathrm{NH}\right)$, 4.49-4.42 (1H, m, CH), $4.12\left(2 \mathrm{H}, \mathrm{d}, J 4.4 \mathrm{~Hz}, \mathrm{CH}_{2}\right), 3.65\left(3 \mathrm{H}, \mathrm{s}, \mathrm{OCH}_{3}\right), 1.37(3 \mathrm{H}, \mathrm{d}, J 7.4 \mathrm{~Hz}$, $\mathrm{CH}_{3}$ ). Anal. Calcd. For $\mathrm{C}_{20} \mathrm{H}_{21} \mathrm{~N}_{3} \mathrm{O}_{5}$ (383.4): C, 62.65; H, 5.52; N, 10.96; Found C, 62.61; H, 5.39; N, 10.87 .

\section{Acknowledgements}

We thank Prof. E. A. Soliman, Faculty of Science, Ain Shams University for technical support and advice.

\section{References}

1. Hlasta, D. J.; Demers, J. P.; Foleto, B. D.; Fraga-Spano, S.; Guan, J.; Hilligard, J. Bioorg. Med. Chem. Lett. 1998, 8, 1923.

2. Vinšová, J.; Imramovský, A. Ces. Slov. Farm. 2004, 53, 294. 
3. Mukta, J.; Shweta, G.; Diwedi, S. C.; Joshi, S. C.; Singh, R. V.; Bansal, A. Phosphorus, Sulfur, and Silicon and the Related Elements 2004, 179, 1517.

4. Waisser, K.; Matyk, J.; Divišová, H.; Husáková, P.; Kuneš, J.; Klimešová, V.; Kaustová, J.; Möllmann, U.; Dahse, H.; Miko M. Arch. Pharm. Chem. Life Sci. 2006, 339, 616.

5. Macielag, M. J.; Demers, J. P.; Fraga-Spano, S. A.; Hlasta, D. J.; Johnson, S. G.; Kanojia, I. M. J. Med. Chem. 1998, 41, 2939.

6. Waisser, K.; Bureš, O.; Holý, P.; Kuneš, J.; Oswald, R.; Jirásková, L. Arch. Pharm. (Weinheim) 2003, 336, 53.

7. Imramovsky, A.; Vinsova, J.; Ferriz, J. M.; Kuneš, J.; Pour, M.; Dolezal, M. Tetrahedron Lett. 2006, 47, 5007.

8. Machoń, Z.; Wieczorek, Z.; Zimecki, M. Pol. J. Pharmacol. 2001, 53, 377.

9. Regiec, A.; Machoń, Z; Mie_dzybrodzki, R.; Szymaniec, S. Arch. Pharm. Chem. Life Sci. 2006, 339, 401.

10. Yoshida, K.; Wakita, T.; Katsuta, H.; Kai, A.; Chiba, Y.; Takahashi, K.; Kato, H.; Kawahara, N.; Nomura, M.; Daido, H.; Maki, J.; Banba, S. WO Patent No. 073165, 2005.

11. Flitter, W. D.; Garland, W. A.; Irwin, I. U.S. Patent 6194465, 2001.

12. Kuroda, N.; Hird, N.; Cork, D. G. J. Comb. Chem. 2006, 8, 505.

13. Desai, A. R.; Desai, K. R. ARKIVOC 2005, (xiii), 98.

14. Sahin, G.; Palaska, E.; Ekizoglu, M.; Ozalp, M. Farmaco 2002, 57, 539.

15. Fathalla, W.; Ali, A. I. Heteroatom Chem. 2007, 18, 637.

16. Ali, I. A. I; Al-Masoudi, I. A.; Saeed, B.; Al-Masoudi, N. A.; La Colla, P. Heteroatom Chem. 2005, 16, 148.

17. Khajavi, M. S.; Shariat, S. M. Heterocycles 2005, 65, 1159.

18. Mohapatra, D. K.; Datta, A. Synlett 1996, 1129. 